\title{
Low flow oxygen therapy in infants
}

\author{
A N CAMPBELL, Y ZARFIN, M GROENVELD, AND M H BRYAN \\ Division of Neonatology, The Hospital for Sick Children, Toronto, Canada
}

SUMMARY Fifty one infants who were oxygen dependent after treatment for neonatal respiratory disease were entered into a study programme where $100 \%$ oxygen was delivered at low flow through a nasal catheter. Thirty five $(69 \%)$ of the infants were discharged home and the remainder were either discharged to a convalescent hospital or back to their peripheral referring hospital. Excluding repeat admissions for monitoring or for the treatment of acute infections, 2760 hospital days (79 days/patient) were saved, representing a financial saving of $\$ 11990$ ( $£ 6500)$ per treated infant. A home low flow oxygen therapy programme has benefits to the infant/parent relationship, provides a more constant flow of oxygen than conventional methods, and the early hospital discharge represents a considerable financial saving.

Modern treatment of newborn infants with respiratory distress syndrome (RDS) has resulted in a notable decrease in mortality. ${ }^{1}$ Although improved methods of mechanical ventilation have contributed to this achievement, up to $20 \%$ of the ventilated survivors have residual lung disease or bronchopulmonary dysplasia (BPD), ${ }^{2}$ and are often oxygen dependent for prolonged periods, requiring extended hospital stay. Other less common lung diseases in newborn infants requiring long term oxygen therapy include the Wilson-Mikity syndrome, meconium aspiration, and lung hypoplasia. Treatment with supplementary oxygen, essential for respiratory recovery as well as for growth and development, may seriously inhibit bonding between the affected infants and their parents ${ }^{2}$ a process already delayed by the initial need for intensive care. ${ }^{3}$ The use of low flow $100 \%$ oxygen $\left(\mathrm{LFO}_{2}\right)$ delivered by nasal catheter to these infants, allows the development of closer physical relationships in hospital, earlier discharge on a home oxygen therapy programme, and reduction in overall medical costs.

\section{Methods}

After transfer from the neonatal intensive care unit (level III) to an intermediate unit (level II), infants requiring supplementary oxygen for residual lung disease are placed on $100 \% \mathrm{LFO}_{2}$ delivered by a nasal catheter, rather than by the conventional delivery of $\mathrm{O}_{2}$ into the hood or isolette. This method of oxygen delivery is possible when oxygen requirements are less than $55 \%$ and the infant weighs more than $1500 \mathrm{~g}$.

A feeding catheter ( $8 \mathrm{FG}$ ) is inserted $1-2 \mathrm{~cm}$ into one naris and secured by tape (Fig. 1). Oxygen $(100 \%)$ is delivered from a wall source via a low flow meter (0-2 l) and $\mathrm{O}_{2}$ tubing attached securely to the nasal catheter. Oxygen requirements are determined individually in each infant, using transcutaneous oxygen tension $\left(\mathrm{T}_{\mathrm{c}} \mathrm{Po}_{2}\right)$ monitoring observed over 1-2 hours of sleep and activity, until a stable oxygen flow maintains the $\mathrm{T}_{\mathrm{c}} \mathrm{Po}_{2}>50 \mathrm{mmHg}$ in all behavioural states. As the infant is breathing predominantly room air, the exact amount of $100 \%$ oxygen inspired from the nasal catheter depends on the infant's tidal volume and frequency (minute ventilation) and the severity of the underlying lung disease. Therefore the actual inspired oxygen concentration $\left(\mathrm{F}_{\mathrm{r}} \mathrm{O}_{2}\right)$ is unknown. The introduction of a pressure manometer (Bird) in the circuit line is an additional safety feature (Fig. 1). The pressure for each patient is dependent on the flow, and a baseline recording is made when the oxygen flow is known to be completely unobstructed. Any notable alterations in this level will indicate interruption to flow (low reading) or an obstruction to flow (high reading). Nasal catheter changes are done using alternate nostrils every three days.

For those infants weighing more than $2500 \mathrm{~g}$ who are still oxygen dependent on the $\mathrm{LFO}_{2}$ system but are otherwise medically stable and are being breast or bottle fed, consideration is given to discharge on low flow oxygen, either home or to their referring hospital. Other important criteria for discharge include acceptance by the parents, a family physician/ paediatrician who is willing to assist in the home or hospital programme, a satisfactory assessment of the home by a health visitor, and a home with more than one resident adult. If all these criteria cannot be met, 


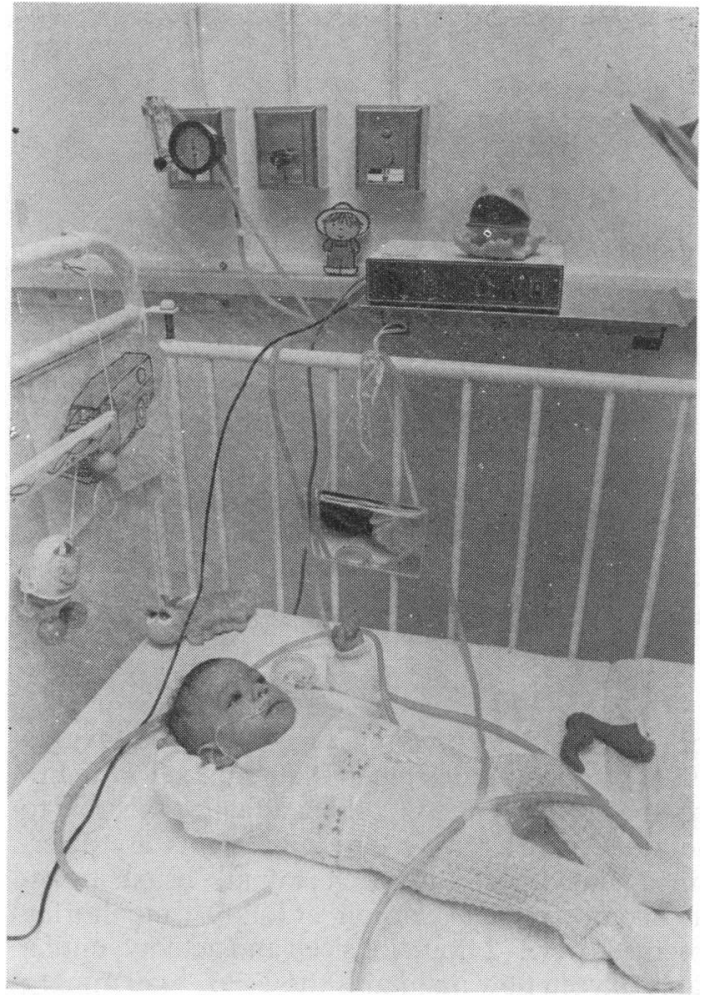

Fig. 1 Infant with nasal oxygen cannula in place together with the $\mathrm{LFO}_{2}$ monitoring equipment. The low flow meter and pressure manometer are shown on the wall (left) conected to the nasal catheter by rubber tubing.

transfer to a local children's convalescent hospital is considered.

If the criteria are met, a two week instruction period for the parents is given by the ward nurses and respiratory technologists. At discharge, the infant patients are transferred by ambulance to the home or hospital, accompanied by a nurse and a respiratory technologist who ensures that the $\mathbf{L F O}_{2}$ system is working and properly adjusted in the home and again instructs the parents. Oxygen is supplied from large cylinders that will last a minimum of three weeks, according to the flow used. Replacement cylinders are obtained by the family direct from the suppliers. For discharge to referring hospitals, the nurse and respiratory technologist accompany the patient and instruct the hospital personnel, supplying and setting up the low flow oxygen meter and the low flow oxygen circuit.

Infants discharged home, or to a peripheral or convalescent hospital are readmitted monthly for 48 to 72 hours to reassess and adjust with $\mathrm{T}_{\mathrm{c}} \mathrm{PO}_{2}$ monitoring, the $\mathrm{LFO}_{2}$ requirements.

Parents are carefully instructed in the symptoms of hypoxia (cyanosis, tachypnoea, apnoea, or signs of a respiratory infection) before discharge, and are asked to seek immediate medical advice for prompt admission of the infant to the hospital if these symptoms occur.

\section{Results}

Over a three year period to June 1982, 51 infants were discharged on $\mathrm{LFO}_{2}$, ranging from 0.05 to $0.5 \mathrm{l} / \mathrm{min}$. Thirty five infants went home, 7 went to the local children's convalescent hospital, and 9 to their peripheral referral hospital. Of the 51 infants discharged, $20(39 \%)$ lived outside Toronto and up to a distance of $1800 \mathrm{~km}$. Table 1 lists the respiratory diagnoses, gestational ages, birthweights, lengths and types of respiratory support in hospital before the introduction of $\mathbf{L F O}_{2}$. Although the most common lung condition was BPD $(n=38)$, there were 8 infants with Wilson-Mikity syndrome, two with pulmonary hypoplasia, two with severe meconium aspiration, and one with eventration of the diaphragm (Table 1). Most infants were placed on $\mathrm{LFO}_{2}$ between weight 1500 and $2500 \mathrm{~g}$ (Fig. 2).

The age at discharge and cessation of oxygen therapy (assessed in hospital with $\mathrm{T}_{\mathrm{c}} \mathrm{Po}_{2}$ monitoring) is shown in Table 2. The mean duration of $\mathrm{LFO}_{2}$ therapy for the 35 infants treated at home ranged from $0 \cdot 5$ to 36 weeks (mean $14 \cdot 1$ weeks).

Of the 35 infants treated at home, 34 were weaned

Table 1 Clinical data of 51 infants treated with low flow oxygen. Values are mean (range)

\begin{tabular}{|c|c|c|c|c|c|c|}
\hline Diagnosis & $N o$ & $\begin{array}{l}\text { Gestational age } \\
\text { (wks) }\end{array}$ & $\begin{array}{l}\text { Birthweight } \\
(k g)\end{array}$ & Days on IPPV & Days on CPAP & Days in head box \\
\hline $\begin{array}{l}\text { Bronchopulmonary dysplasia } \\
\text { Wilson-Mikity syndrome } \\
\text { Hypoplastic lungs } \\
\text { Other* }\end{array}$ & $\begin{array}{r}38 \\
8 \\
2 \\
3\end{array}$ & $\begin{array}{l}30 \cdot 2(24-37) \\
28 \cdot 3(27-31) \\
32(28-36) \\
35(32-40)\end{array}$ & $\begin{array}{l}1 \cdot 28(0 \cdot 6-2 \cdot 3) \\
1 \cdot 17(0 \cdot 7-1 \cdot 5) \\
1 \cdot 54(1 \cdot 1-1 \cdot 9) \\
1 \cdot 7(1 \cdot 2-4 \cdot 2)\end{array}$ & $\begin{aligned} 16 & (2-76) \\
2 & (0-7) \\
14 & (0-28) \\
3 & (0-6)\end{aligned}$ & $\begin{array}{l}7(0-30) \\
4(0-17) \\
3(0-6) \\
7(0-21)\end{array}$ & $\begin{array}{l}44(2-134) \\
43(21-68) \\
37(11-64) \\
33(18-52)\end{array}$ \\
\hline
\end{tabular}

*Meconium aspiration (2), eventration of diaphragm (1).

IPPV $=$ intermittent positive pressure ventilation; CPAP $=$ continuous positive airway pressure. 


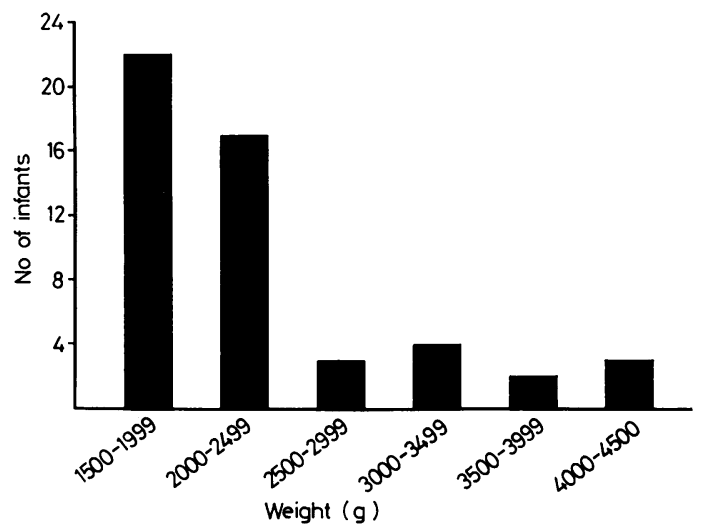

Fig. 2 Weight of the 51 infants when begun on low flow oxygen therapy.

Table 2 Treatment of 51 infants on low flow oxygen $\left(\mathrm{LFO}_{2}\right)$

\begin{tabular}{lcll}
\hline Diagnosis & No & $\begin{array}{l}\text { Age discharged } \\
\text { on LFO } \\
\text { Mean (range) } \\
(w k s)\end{array}$ & $\begin{array}{l}\text { Age when } \mathrm{LFO}_{2} \\
\text { ceased } \\
\text { Mean (range) } \\
(w k s)\end{array}$ \\
\hline $\begin{array}{l}\text { Bronchopulmonary } \\
\text { dysplasia }\end{array}$ & 38 & $18 \cdot 1(5-59)$ & $29.9(9-75)$ \\
$\begin{array}{l}\text { Wilson-Mikity } \\
\text { syndrome }\end{array}$ & 8 & $15 \cdot 3(9-25)$ & $29.3(15-45)$ \\
$\begin{array}{l}\text { Hypoplastic lungs } \\
\text { Other }\end{array}$ & 2 & $\begin{array}{c}8 \\
(5-11)\end{array}$ & $\begin{array}{l}22.5(20-23) \\
21.6(10-32)\end{array}$ \\
\hline
\end{tabular}

from $\mathrm{LFO}_{2}$ and 32 survive. One infant was readmitted to hospital 4 days after discharge home on $\mathrm{LFO}_{2}$ because the family failed to manage. One infant with BPD was successfully weaned from therapy at age 9 months after 23 weeks of home oxygen, but died suddenly at home 5 weeks later. The necropsy showed residual BPD and bronchopneumonia. A second death occurred suddenly at home at age 5 months -5 weeks after cessation of $\mathrm{LFO}_{2}$ therapy. A necropsy showed Wilson-Mikity syndrome. Eight infants required readmission during home therapy for management of upper respiratory infections on 12 separate occasions, representing 146 days of additional hospital treatment. One of those infants with severe BPD, treated initially for 59 weeks in hospital, followed by 16 weeks of $\mathrm{LFO}_{2}$ at home, died on a ventilator in hospital with bronchopneumonia at age 18 months, never having been weaned from oxygen therapy. All infants under 36 weeks' gestation had their fundi examined for evidence of retrolental fibroplasia (RLF), monthly during their initial illness and subsequently on readmission. No baby developed RLF or had any progression while on home oxygen therapy.

\section{Discussion}

Pinney and Cotton in $1976^{4}$ first described the successful treatment of oxygen dependent infants at home on nasal catheter $(100 \%)$ oxygen. Oxygen delivered in this manner using $\mathrm{T}_{\mathrm{C}} \mathrm{Po}_{2}$ monitoring has been shown to achieve a more stable infant oxygenation when compared with conventional $\mathrm{O}_{2}$ delivery into the incubator or blown over the face. ${ }^{5}$ Improved weight gain has also been achieved. ${ }^{6}$ This $\mathrm{LFO}_{2}$ method allows for increased contact between the infant and his parents and caretakers while in hospital, as well as nursing in an open basinette or cot rather than the enclosed incubator environment. The improved infant/parental contact possible when the baby is cared for by the parents at home, despite oxygen dependency, at the least allows more positive relationships to develop within the family. This is particularly important for parents who live outside the city limits for whom visiting is difficult.

Home oxygen management does entail some risk to the infant. Not only must the parents be enthusiastic about the home programme, they must also have repeated and careful practical instruction, show ease in handling the equipment, and recognise infant hypoxia in order to increase the oxygen delivery before they contact their physician or the hospital. None of our parents wished to abandon the programme, once initiated, and return their infant to the hospital, but many expressed gratitude for the monthly admissions of 2-3 days for reassessment of the infant's oxygen needs and well being. These admissions gave many families a respite from the constant vigilance needed in looking after the oxygen dependent infant. Because of these demands, readily accepted by the parents, the presence of two adult family members in the home eases the burden of responsibility.

One of our infants died in hospital, with severe BPD and respiratory failure, and the death was expected by the parents and ourselves. However, two infants died, both with residual chronic lung disease, five weeks after the home oxygen programme ended. Since these deaths, infants now remain on home apnoea monitors for two months after oxygen therapy has ceased.

The introduction of portable oxygen packs has allowed infants to be driven by car to and from the hospital for their assessments, decreasing the need for ambulance transport, and in addition allowing the family short excursions from their homes for pleasure outings.

Another benefit of the home oxygen programme is its cost effectiveness. ${ }^{7}$ In Ontario, Canada, the average daily cost of an inpatient at the time of 
treatment was $\$ 156$ (£80). Assuming that the time spent on oxygen at home would have been the same as if the infant had remained in hospital, there was a saving of 3066 hospital days among the 35 home treated babies. Deducting the 146 days required for hospital treatment during acute infections, and the $\mathbf{2 5 0}$ days in hospital for monitoring and general reassessment, the saving was 2760 days. This represents a financial saving of $\$ 416520$ or $\$ 11990$ ( $£ 6500)$ per treated infant. On the other hand, the cost of maintaining oxygen in the home, nasal catheter supplies, etc was an average of $\$ 100(£ 50)$ per month per infant. The benefits to any health care programme, national or individual, are obvious.

A home $\mathrm{LFO}_{2}$ therapy programme has considerable theoretical benefits for the oxygen dependent infant and his family, in allowing normal interpersonal relationships and perhaps enhancing infant growth and development. For the same reasons the $\mathrm{LFO}_{2}$ method is also beneficial in the hospital setting, and ensures a more constant oxygen delivery than conventional methods. The home programme, allowing early discharge from hospital, represents a considerable financial saving.
We thank Keith Matthews (respiratory technologist), Joanne Hunt (continuing care coordinator) and Dianne Hamilton (public health nurse) without whose hard work the home therapy plan would not be possible.

\section{References}

1 Hack M, Fanaroff AA, Merkatz IR. The low-birth-weight infant-evolution of a changing outlook. $N$ Engl $J$ Med $1979 ; 301: 1162-5$.

2 Wung JT, Koons HH, Driscoll JM, James LS. Changing incidence of bronchopulmonary dysplasia. J Pediatr $1979 ; 95: 845-7$.

3 Klaus MH, Kennell JH. Maternal infant bonding. London: CV Mosby, 1976.

4 Pinney MA, Cotton EK. Home management of bronchopulmonary dysplasia. Pediatrics 1976;58:856-9.

5 Philip AGS, Peabody JL, Lucey JF. Transcutaneous $\mathrm{PO}_{2}$ monitoring in the home management of bronchopulmonary dysplasia. Pediatrics $1978 ; 61: 665-7$.

6 Cox MA, Cohen AJ, Slavin RE, Epstein MF. Improved growth in infants with bronchopulmonary dysplasia treated with nasal cannula oxygen (Abstract). Pediatr Res 1979;13:1001.

7 Donn S. Cost effectiveness of home management of bronchopulmonary dysplasia (letter). Pediatrics 1982;70: $330-1$.

Correspondence to $\operatorname{Dr}$ A N Campbell, Department of Paediatrics, Sunderland District General Hospital, Kayll Road, Sunderland SR4 7TP.

Received 3 June 1983 